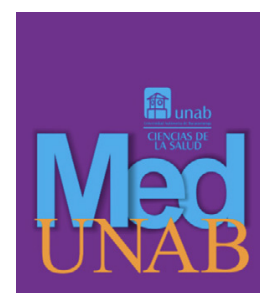

REVISTA DE LA FACULTAD

DE CIENCIAS DE LA SALUD

\title{
Comprensión del vínculo persona mayor-mascota como alternativa para la promoción de la salud
}

Understanding the elderly-pet bond as an alternative for health promotion

Compreensão do vínculo pessoa idosa-animal de estimação como alternativa de promoção da saúde

Marleny Zapata-Herrera, Enf., Mg., Esp. ${ }^{1}$ (D), Catalina María Sanmartín-Laverde, Enf., Mg., Esp. ${ }^{2}$ DD, Teresa Nury Hoyos-Duque, Enf., Mg., Esp. ${ }^{3}$ (D)

1. Enfermera, Magíster En Salud Colectiva, Especialista En Salud Ocupacional. Facultad de Enfermería Universidad de Antioquia, Medellín, Colombia.

2. Enfermera, Magíster En Salud Pública, Especialista En Salud Ocupacional. Facultad de Enfermería Universidad de Antioquia, Medellín, Colombia.

3. Enfermera, Magíster en Educación y Desarrollo Humano, Especialista en Docencia Universitaria. Facultad de Enfermería Universidad de Antioquia, Medellín, Colombia.

Correspondencia. Marleny Zapata Herrera. Facultad de Enfermería Universidad de Antioquia. Calle 64 \# 5309 Medellín, Antioquia. Email. marleny.zapata@udea.edu.co

\section{INFORMACIÓN DEL ARTÍCULO:}

Artículo recibido: 14 de diciembre de 2020

Artículo aceptado: 17 de junio de 2021

DOI: https://doi.org/10.29375/01237047.4056

Cómo citar. Zapata-Herrera M, Sanmartín-Laverde CM, Hoyos-Duque TN. Comprensión del vínculo persona mayor-mascota como alternativa para la promoción de la salud. MedUNAB. 2021; 24(2):193-202 doi: https://doi. org/10.29375/01237047.4056

\section{RESUMEN}

Introducción. el aumento de personas mayores en Colombia sumado a la pobreza y a la falta de soporte familiar y redes de apoyo para estas personas constituye un reto para el Estado. Ante esta problemática se exploran alternativas como la influencia de las mascotas en su salud. El objetivo de este manuscrito es comprender el vínculo persona mayor-mascota en la vida cotidiana y las implicaciones para su salud. Metodología: se trata de un estudio cualitativo con perspectiva etnográfica focalizada. Se realizaron 14 entrevistas semiestructuradas y observación a mayores de 60 años que conviven con mascotas en Medellín-Colombia. Participaron 8 mujeres, 6 hombres, entre 60 y 85 años. Mascotas: 17 perros y 7 gatos. Resultados: 
emergieron cuatro categorías principales: la mejor compañía, un día con la mascota, relación familia-mascota y beneficios de la mascota para la promoción de la salud de los mayores. Discusión: para los mayores lo más satisfactorio de tener mascota es su compañía, comparable a la de un hijo o un ser querido, seguido de la posibilidad que les brinda de expresar emociones placenteras, aumentar su interacción social y sentirse útiles y activos; beneficios que trascienden al resto de la familia. Conclusiones: el vínculo persona mayor-mascota es un coadyuvante para la promoción de la salud.

Palabras Claves:

Anciano; Mascota; Comprensión; Vínculo Humano-Animal; Promoción de la Salud.

\section{ABSTRACT}

Introduction. The increase in the number of elderly people in Colombia, coupled with poverty and the lack of family care and support networks for these people, constitutes a challenge for the State. Faced with this problem, alternatives such as the influence of pets on their health are being explored. The purpose of this article is to understand the elderly-pet bond in everyday life and the implications for their health. Methodology. This is a qualitative study with a focused ethnographic perspective. Fourteen semi-structured interviews and observation were conducted with people over 60 years of age who live with pets in Medellín, Colombia. Participants included 8 women and 6 men between the ages of 60 and 85. Pets: 17 dogs and 7 cats. Result. Four main categories emerged: the best company, a day with the pet, family-pet relationship and benefits of the pet in promoting the health of the elderly. Discussion. For the elderly, the most satisfying aspect of having a pet is its companionship, comparable to that of a child or loved one, followed by the possibility it gives them to express pleasant emotions, increase their social interaction and feel useful and active; benefits that transcend to the rest of the family. Conclusions. the elderly person-pet bond is an adjuvant for health promotion.

Keywords:

Elderly; Pet; Understanding; Human-Animal Bond; Health Promotion.

\section{RESUMO}

Introdução. $\mathrm{O}$ aumento da população idosa na Colômbia, aliado à pobreza e à falta de apoio familiar e de redes de apoio a essas pessoas, constitui um desafio para o Estado. Diante dessa problemática, são exploradas alternativas tais como a influência dos animais de estimação em sua saúde. Metodologia. Este é um estudo qualitativo com uma perspectiva etnográfica focalizada. Foram realizadas 14 entrevistas semiestruturadas e observação de pessoas com mais de 60 anos que vivem com animais de estimação em Medellín-Colômbia. Participaram 8 mulheres, 6 homens, com idade entre 60 e 85 anos. Animais de estimação: 17 cães e 7 gatos. Resultados. Emergiram quatro categorias principais: a melhor companhia, um dia com o animal de estimação, relação família-animal de estimação e benefícios do animal de estimação para a promoção da saúde do idoso. Discussão. Para os idosos, o mais gratificante em ter um animal de estimação é a sua companhia, comparável à de uma criança ou de um ente querido, seguida da possibilidade que lhes oferece de expressar emoções agradáveis, aumentar a sua interação social e sentir-se úteis e ativos; benefícios que transcendem para o resto da família. Conclusões. $\mathrm{O}$ vínculo pessoa idosa-animal de estimação é coadjuvante da promoção da saúde.

Palavras-chave:

Pessoa idosa; Animal de estimação; Entendimento; Vínculo Humano-Animal; Promoção da saúde.

\section{Introducción}

El vínculo, o apego humano-animal, hace referencia a la relación íntima que establecen la personas con especies animales como los perros y gatos (1). Mascota, en inglés pet, se origina del francés petit o pequeño, un término que hace referencia a que hacia el año 1500 a los animales de compañía se les trataba con indulgencia, como favoritos, igual que a un niño (2). La convivencia con mascotas se incorpora cada vez más a las familias y en regiones como Norteamérica y 
Europa se ha estudiado el vínculo humano-animal en las diferentes etapas del ciclo vital de las personas.

Según la CEPAL (Comisión Económica para América Latina y el Caribe) se considera que la vejez comienza a partir de los 60 o 65 años y, por ley, a las personas en este rango de edad se les asignan denominaciones como "adulto mayor" o "persona adulta mayor" (PM). La vejez y el envejecimiento involucran aspectos fisiológicos, sociales, culturales y políticos (3).

Según la OMS (Organización Mundial de la Salud), se prevé un rápido incremento de la población mayor de 60 años en la región de las Américas (4), que para América Latina y el Caribe estará cercano al $25 \%$ en el año 2050, y será mayor en los próximos decenios, en particular en países de bajos y medianos ingresos (5).

En Colombia el envejecimiento se da en un contexto de vulnerabilidad, pobreza, bajo nivel educativo, precariedad laboral y bajos ingresos (6). Las principales causas de mortalidad en esta población (7) son las complicaciones por enfermedad cardiovascular, osteomuscular y endocrina (8). En relación con la salud mental prevalecen los síntomas depresivos $(41.0 \%)$ y el deterioro cognoscitivo leve $(17.5 \%)$, que se agudizan por el aislamiento y la inactividad (7).

En muchos casos las personas mayores (PM) se ven afectadas por falta de soporte familiar y redes de apoyo, pues algunas familias, por causas económicas, familiares o laborales, optan por institucionalizarlas, lo que lleva a falta de atención, aislamiento social y, en ocasiones, abandono y deterioro de su salud mental (9).

Ante este panorama, agravado por la desprotección del Estado, se considera necesario explorar la relación de las PM con las mascotas, ya que diferentes estudios evidencian beneficios para la salud física y mental y la prevención de enfermedades (10-12). Desde esta perspectiva, el profesional de enfermería y las instituciones de salud pueden considerar a la mascota como una alternativa para el cuidado de la salud de las personas, la familia y la comunidad. En Colombia el estudio de este fenómeno es incipiente, por lo que se plantea el objetivo de comprender el vínculo persona mayor-mascota y sus implicaciones para la salud.

\section{Metodología}

Este estudio se hizo en Medellín (Colombia) y su Área Metropolitana, entre los años 2018-2020, con enfoque cualitativo enmarcado en la etnografía particularista o enfocada (13), donde el investigador participó de la cotidianidad de las personas durante algún tiempo mediante la observación, entrevista y recolección de datos (14) para comprender el vínculo persona mayormascota desde la voz de los participantes (perspectiva emic), y la interpretación y análisis del investigador (visión etic) (13).

Para referirnos a los participantes se usaron los términos "persona mayor" o "los mayores", y para el animal el término "mascota". La población estuvo constituida por PM que convivían con una mascota. Se hizo muestreo intencionado teniendo en cuenta: sexo, tipo de mascota, lugar de residencia y estrato socioeconómico. Para la selección de los participantes se recurrió a los porteros (14) por intermedio de contactos personales y vecinos de las investigadoras.

Los criterios de inclusión fueron: persona mayor de 60 años, capaz de responder la entrevista, que conviviera con una mascota por tiempo superior a 6 meses, con un vínculo de interacción o cuidado de la misma. Los criterios de exclusión fueron: enfermedades agudas y dificultad comunicativa del participante.

La recolección de los datos se inició con tres entrevistas semiestructuradas que hicieron parte de la prueba piloto y cuando se llegó a la entrevista $\mathrm{N}^{\circ} 14$ se obtuvo la saturación de los datos (13). Para las entrevistas se cumplieron los requisitos éticos: consentimiento informado verbal, escrito y grabación en audio, el promedio de duración de cada una fue de 120 minutos, al mismo tiempo se hizo la observación ( 30 horas) en la vivienda para conocer rutinas, dinámica familiar, relación de la PM con la mascota. La entrevista se grabó y se consignó en diarios de campo que aportaron la comprensión de los datos para el análisis. Se hizo análisis y codificación manual de cada una de las entrevistas (trascripción, codificación línea por línea y comparación de códigos de testimonios) (15), luego se compararon con otras entrevistas, así sucesivamente hasta obtener la depuración y el ordenamiento conceptual, que dieron origen a las categorías principales y subcategorías, que dan respuesta a la pregunta del estudio (Tabla 1).

Una vez obtenidos los resultados del estudio se presentaron a pares de investigación de la comunidad académica de la Facultad de Enfermería de la Universidad de Antioquia y se hizo la validación de resultados en un encuentro virtual con los participantes del estudio y otras personas mayores. Este estudio se clasificó de riesgo bajo, según Resolución 00843016 de 1993 del Ministerio de Salud de Colombia. Para su realización se tuvieron en cuenta todos los principios 
éticos y se contó con la aprobación del Comité de Ética de la Facultad de Enfermería de la Universidad de Antioquia.

Tabla 1. Categorías y subcategorías. Comprensión del vínculo persona mayor-mascota como alternativa para la promoción de la salud.

\begin{tabular}{|c|c|}
\hline Categorías & Subcategorías \\
\hline \multirow{3}{*}{ La mejor compañía } & $\begin{array}{l}\text { 1. La llegada de la } \\
\text { mascota }\end{array}$ \\
\hline & 2. Estar ahí \\
\hline & 3. Como un hijo \\
\hline \multirow{2}{*}{$\begin{array}{l}\text { Un día de la persona mayor con } \\
\text { la mascota }\end{array}$} & $\begin{array}{l}\text { 4. Motivo para levan- } \\
\text { tarse }\end{array}$ \\
\hline & $\begin{array}{l}\text { 5. Cuidados con la } \\
\text { mascota }\end{array}$ \\
\hline \multirow[b]{2}{*}{ Relación familia- mascota } & 6. Convivencia familiar \\
\hline & $\begin{array}{l}\text { 7. Situaciones difíciles } \\
\text { con la mascota }\end{array}$ \\
\hline \multirow{3}{*}{$\begin{array}{l}\text { Beneficios de la mascota para } \\
\text { la promoción de la salud de las } \\
\text { personas mayores }\end{array}$} & $\begin{array}{l}\text { 8. Expresión de emo- } \\
\text { ciones }\end{array}$ \\
\hline & 9. Actividad física \\
\hline & 10.Interacción social \\
\hline
\end{tabular}

\section{Resultados}

Los participantes fueron 14, con edades entre 60 y 85 años, predominantemente mujeres: $57.1 \%$; estado civil: casados $57.1 \%$ y separados $28.5 \%$; estrato socioeconómico 3: medio-bajo 64.5\%; nivel de educación secundaria incompleta: $78.5 \%$. Los familiares de los participantes fueron 42, predominó la familia nuclear: $57.1 \%$, seguida de la extensa $28.5 \%$. La mayoría de los participantes convivía con más de dos mascotas: $71.4 \%$, generalmente perros: $70.8 \%$, y gatos: $29.2 \%$, con un promedio de tenencia de 10 años.

Los resultados del estudio estuvieron representados en cuatro categorías principales y subcategorías que emergieron desde la voz de los participantes (Tabla 1).

\section{La mejor compañía}

Para la PM lo más satisfactorio de tener una mascota en el hogar es la compañía que esta le brindaba y el vínculo que establece con ella, similar al que se forma con un hijo o un ser querido. Esta categoría se explica en tres subcategorías: llegada de la mascota; estar ahí y como un hijo.

\section{La llegada de la mascota}

Las mascotas llegan por intermedio de un hijo, nieto $\mathrm{u}$ otro familiar, al principio las PM expresaban negación y resistencia, pero finalmente la acogían y asumían su cuidado como lo expresan estos testimonios:

"Cuando se consiguió ese novio, le trajo un perrito y lo tuve que aceptar" (E7, p10).

"Primero me tuvieron que consentir a mí, porque nosotros ya habíamos tenido otra mascota, pero yo no quería más perros... Me echaron carreta, me echaron el cuento, hasta que me convencieron" (E8, p1).

\section{Estar ahí}

Las mascotas acompañaban a las PM en todos los momentos y espacios cotidianos, siempre querían estar junto a ellas, estaban pendientes de las rutinas y actividades que realizaban. Les seguían los pasos.

"Ellas ven cuando uno está empacando el bolso, vea, no se acuestan, son detrás de mí, si voy a la pieza, si voy a la cocina, como quien dice: no me vaya a dejar" $(\mathrm{E} 4, \mathrm{p} 5)$.

"Este animalito es detrás de mí, si voy a la tienda se va para el mirador (balcón) a esperarme que llegue y me asome, ahí mismo ladra, y cuando abro está esperándome" $(\mathrm{E} 2, \mathrm{p} 5)$.

La compañía permanente de la mascota apoyaba los momentos de soledad y la necesidad de comunicación con el otro.

"Son una compañía, tiene uno con quien hablar, jugar, con quien salir, porque la mayoría de los hijos se fueron... Uno no se siente solo" (E4, p10).

"Al menos puedo hablar con él en esta soledad, tengo a Coco, eso tiene su lado positivo, si no estuviera aquí no tenía con quién mover la boca, la soledad pega duro y son muchos los días de soledad" (E10, p4).

\section{Como un hijo}

Los mayores crearon sentimientos de apego a la mascota, tan significativos, que la comparaban con un ser querido, "un hijo o niño pequeño". Ellos tenían 
claro que no era lo mismo que un hijo, pero les generaba afecto y ternura.

"El día que nos toque dejarla sola ¿qué vamos a hacer? Es como cuando estábamos solos que teníamos un hijo enfermo y otro pequeño, y le decía yo a mi esposa, ¿con quién vamos a dejar el niño?” (E2,P9).

"Uno se apega mucho, al llegar y no escucharla uno dice ¿dónde está loris?, yo la llamo... Se siente ahí mismo como ese vacío, uno se apega mucho a los animales." $(\mathrm{E} 5, \mathrm{p} 3)$.

\section{Un día de la persona mayor con la mascota}

La mascota estimulaba a las PM a realizar actividades relacionadas con su cuidado que se volvían rutinarias. Así mismo, las obligaba a establecer horarios y un orden para ejecutarlas que favorecía su capacidad funcional y las mantenía activas. Esta categoría está soportada en dos subcategorías: motivo para levantarse y cuidados con la mascota.

\section{Motivo para levantarse}

La mascota era un estímulo para que la PM se levantara en la mañana en un horario habitual; el comportamiento animal (ladridos, quejidos, movimiento de manos) obligaba al mayor a levantarse de la cama para sacarla a satisfacer sus necesidades fisiológicas, suministrarle la alimentación o salir a caminar.

"Hay un motivo para levantarse, para bañarse tempranito a la misma hora de sacarlos, porque ellos ya tienen ese horario" (E1, p9).

"Cuando estoy acostado un fin de semana y duermo hasta tarde, Bruno se preocupa ¿por qué no se ha levantado? Me lame la cara, como quien dice «levántate pues» y empieza a chillarme" (E6, p13).

\section{Cuidados con la mascota}

Durante el día la mascota demandaba de las PM tareas básicas de cuidado que generaban beneficio mutuo y satisfactorio. La mascota, por su parte, contaba con el compromiso de los mayores de cuidarla y satisfacer sus necesidades, y ellos, además de comprometerse, se mantenían ocupados y se sentían útiles.

"Una de las primeras actividades es salir a caminar dos, tres veces al día con ella, después se le da la primera comida y sigue todo normal hasta por la tarde que salgo con ella" (E13, p3).

"No cuidarla bien es que no la aseen o no le tengan el agüita, el cuido y la coca donde se desocupa (hace necesidades fisiológicas)" (E11, p16).

\section{Relación familia-mascota}

La relación de la PM con la mascota se manifestaba de manera positiva en los demás miembros de la familia. Esta categoría se describe en dos subcategorías: convivencia familiar y situaciones difíciles con la mascota.

\section{Convivencia familiar}

La presencia de la mascota estimulaba la convivencia familiary ayudaba a afrontarlos problemas cotidianos, era una especie de mediador que favorecía la comunicación y la paz del hogar. Los mayores expresaron cambios positivos en la familia y el comportamiento de los hijos.

“...También ha sido el mediador de toda la familia, que no peleemos, que mantengamos la armonía familiar, cuando llegaron los gatos toda esa discusión y todo eso, desapareció" (E7, p3).

"La primera mascota llegó cuando murió mi papá, yo ya me había separado y mi papá fue la figura paterna de mi hija; ella tenía 7 años, se puso muy rebelde, indisciplinada en el colegio y la psicóloga me recomendó que le consiguiera una mascota que ella pudiera abrazar y apechichar (dar abrazos, caricias y otras muestras de cariño) y una amiga nos regaló a Paquita y mi hija cambió considerablemente" (E10, p3).

\section{Situaciones difíciles con la mascota}

Los participantes manifestaron que una situación difícil era cuando tenían que separarse de su mascota, si se trataba de periodos cortos buscaban a algún familiar o amigo que tuviera empatía con ella pues sabían que se quedaría en buenas manos. Pero la situación más triste se daba por enfermedad y muerte de la mascota, toda vez que la consideraban una experiencia que afectaba su vida por el vínculo que habían establecido con ella: la consideraban un miembro más de la familia:

"Entonces las encontré (las hijas) chillando... La cosa más horrible, en el desespero que ellas se querían morir; esa experiencia fue horrible (la muerte), con sólo recordarlo me da dolor de cabeza" (E7, p20). 
"Lo más difícil es cuando están viejitos; por ejemplo, hace poco, el gato, el siamés, ese duró 17 años, le tuvimos que hacer la eutanasia, aquí sentados, y ella (la hija) cargándolo y llorando" (E9, p18).

\section{Beneficios de la mascota para la promoción de la salud de las personas mayores}

Esta categoría está soportada en tres subcategorías: expresión de emociones, actividad física e interacción social.

\section{Expresión de emociones:}

Los mayores consideraron que lo más satisfactorio de tener una mascota en el hogar era la alegría y felicidad que producían.

"Es sobre todo emocional, pues como te digo, se siente la presencia y ella le da a uno mucha alegría por las cosas que hace es la alegría de la casa" (E5 p6).

"Un médico le decía a Flor que este animalito era como una bendición, una felicidad, y que lo íbamos a ver, y no lo creíamos... Pero es un gusto tener ese animal, es cierto. La felicidad de uno, la felicidad mía, la felicidad de la casa" (E2, p11).

Así mismo, los mayores comentaron que en momentos de tristeza, ira y angustia por asuntos laborales y familiares la mascota se convertía en un apoyo y una compañía que ayudaban a superar esas emociones y devolver la tranquilidad.

"Si estoy triste o llorando, él percibe esa energía y está siempre ahí conmigo. Por ejemplo, yo puedo estar en el baño llorando y él está en la puerta esperándome y siento sus paticas en la puerta, angustiado" (E10, p6).

"Manejar gente es muy verriondo, que un cliente me diga que «usted es un ladrón» yo llego aquí con esa rabia, pero cuando me reciben los perros, se me cambia el genio y dejo lo que me dijo ese cliente y lo que me da es risa" (E6, p12).

\section{Actividad física}

Tener mascotas, especialmente perros, era para los mayores un motivo para salir a caminar en horarios regulares y satisfacerles una de sus necesidades, actividad que con el tiempo se les volvía placentera y benéfica para la salud.

"A veces él me pide una salida, entonces yo le digo: enseguidita lo saco, y él me persigue, llora, yo sé que es una necesidad que yo lo saque, él se comunica y uno aprende a identificar esos gestos" (E10, p8).

"Uno por ellas tiene que salir, haga sol o llueva, entonces uno se arregla y a caminar... Eso le sirve a uno, cualquier caminadita baja un poquito de calorías y yo soy propensa a ser obesa" (E4, p9).

\section{Interacción social}

Las mascotas promovían y facilitaban las relaciones con otras personas en el vecindario, parques o grupos sociales, alrededor de ellas siempre hay un motivo para conversar y compartir experiencias.

"Cuando va uno a los parques y se encuentra a alguien que también tiene perro, uno entabla conversación, se hablan bobadas, muchas pendejadas" (E8, p30.)

"Otras personas se han acercado más por el hecho de tener gatos y uno ha hecho relaciones en el club de vida, por ejemplo: yo también tengo un gato ¿qué le mando?, ¿cómo es el tuyo? ¿que cómo es el mío?” (E7,p17).

Testimonios: $\mathrm{E}$ : $\mathrm{N}^{\mathrm{o}}$ de entrevista del participante; $\mathrm{p}$ : página de la entrevista.

\section{Discusión}

\section{La mejor compañía}

Las mascotas llegan al hogar de los mayores a través de terceros (hijos, nietos, sobrinos u otros), no por iniciativa propia (16). Las PM son conscientes del compromiso que implica tenerlas y cuidarlas, son renuentes a tenerlas (17) pero una vez en el hogar se va creando una relación estrecha entre ellas que los expertos califican como peculiar y beneficiosa. Las mascotas están ahí y acompañan a las PM siempre, con su compañía se produce un efecto de intimidad compartida y espontánea, acompañado de caricias y juegos (18).

La intimidad y la compañía se soportan en la capacidad de comunicación entre humanos y animales (19); las PM buscan su compañía por sentir la responsabilidad del cuidado y afecto, similar al rol de un miembro de la familia (18). Las PM se sienten satisfechas con las 
mascotas actúan como mediadoras para afrontar los problemas familiares con resultados positivos de unión, cooperación y mejores relaciones interpersonales. Las mascotas, con las rutinas que implican, se integran a la familia y ayudan a moldearla, al tiempo que favorecen su estructura jerárquica y el cumplimiento de normas, elementos que son clave en la educación de niños, jóvenes y otros miembros de la familia (17).

seguridad, encontrado en este estudio.

El apego o el vínculo se pueden revisar desde la teoría del apego de Bowlby (24), los seres humanos están predispuestos, como otros animales, a buscar y mantener apego o contacto físico y emocional con determinadas figuras familiares que les brindan protección física y psicológica.

El vínculo humano-animal tiene gran aceptación en la vida de las personas, con lazos de amor, lealtad y dedicación incondicional (1), los mayores le confieren a la mascota un rol y lugar significativo e importante dentro del grupo familiar, al punto de referirse a ella como mi hijo, mi nieto o el niño peludo (25). Con este vínculo los mayores se sienten importantes, con motivos para vivir (1). Un estudio para medir el apego en adultos dueños de mascotas, en diferentes grupos de edad, concluye que las PM, en especial las mujeres, presentan mayor grado de apego y, que a mayor apego con la mascota, mayores son los efectos benéficos para su salud (26).

\section{Un día de la persona mayor con la mascota}

La capacidad funcional en los adultos mayores está dada por el grado de independencia para las actividades diarias en el hogar y desempeño de roles (27). Algunos estudios demuestran que los perros son la razón para que los mayores se levanten en la mañana en un horario habitual (28), adquieren autodisciplina para madrugar y salir a caminar con ellos al punto que ni las adversidades del clima se los impide (29). Durante el día la mascota demanda tareas rutinarias como la alimentación, caminatas y la satisfacción de sus necesidades fisiológicas, actividades que benefician a los mayores en la medida en que los mantiene ocupados, activos y con la responsabilidad de planear el día a día para emprender tareas físicas y cognitivas que impactan positivamente su salud mental y su capacidad funcional $(30,31)$.

\section{Relación familia-mascota}

La relación humano-animal se incorpora cada vez más a la dinámica y convivencia como parte de la vida familiar (32). Los mayores reconocen que las
Las PM dicen que las experiencias más difíciles de la convivencia con las mascotas son la separación y la muerte, expresan inquietud y temor de perderlas (31) y equiparan estas pruebas con la separación de un niño pequeño. La muerte se convierte en un evento que los mayores recuerdan como algo "horrible", comparable con la de un ser querido (30) que produce dolor, tristeza, llanto y soledad; expresiones que trascienden a la familia (32). La pérdida evidencia en los participantes la capacidad de apego, de la que no son conscientes y que implica la elaboración de un duelo (33).

\section{Beneficios de la mascota para la promoción de la salud de las personas mayores}

El vínculo persona mayor-mascota contribuye a que las PM alcancen un mejor control sobre su estado de salud y bienestar físico, mental y social, toda vez que favorece la expresión de emociones, la actividad física y la interacción social. Las emociones comúnmente expresadas por los mayores son conocidas como básicas o primarias (tristeza, asombro, alegría, asco, ira y miedo), su estudio ayuda a entender aspectos prácticos de la interacción social, relaciones afectivas, comunicación, aprendizaje y tipos de inteligencia, herramientas para el diagnóstico y la terapia emocional de las personas (34).

Los mayores opinan que el gran beneficio del vínculo PM-mascota se relaciona con la tranquilidad, seguridad, alegría, felicidad (29) y el control de emociones como la tristeza, la ira y la angustia que mejoran el estado anímico y reducen el estrés (31). Este beneficio para la salud mental es un factor protector cardiovascular, incluso más fuerte que la misma actividad física (35). No obstante, el estudio de las emociones en PM con tenencia de mascotas requiere mayor evidencia, ya que existen variables sociodemográficas y de personalidad que pueden modificar los resultados (36).

La American Heart Association afirma: "podría ser razonable los beneficios para PM con mascotas, particularmente perros, para la reducción del riesgo de enfermedad cardiovascular (Nivel de evidencia $B$ intermedio) Clase IIb: datos de un único ensayo 
clínico aleatorizado o estudios no aleatorizados)" (37). Al comparar dueños de perros con dueños de gatos, se observa que los primeros tienen menor riesgo cardiovascular por el incremento del número de caminadas, que mejora la actividad física (12\% más activos) (38) y reduce los índices de presión arterial, frecuencia cardiaca y masa corporal (39).

Además del ejercicio físico, el vínculo PM-mascota promueve la interacción con otras personas, las mascotas actúan como un "conector" para una mayor socialización $(31,35)$ y es el perro el que más aporta en ese aspecto (40).

Los resultados de este estudio evidencian que el vínculo PM-mascota es un coadyuvante para la promoción de la salud de las PM en su entorno familiar y comunitario, en esta medida se recomienda que los profesionales del área de la salud lo integren en los planes de cuidado.

\section{Limitaciones}

La mayoría de las investigaciones encontradas sobre el fenómeno de estudio tienen un enfoque predominantemente cuantitativo y se desarrollaron en Norteamérica y Europa, solo se hallaron nueve estudios cualitativos, aspecto que limita una mayor discusión de las categorías emergentes en el contexto de América Latina. Lo anterior motiva a continuar estudiando este fenómeno desde diferentes metodologías y escenarios.

\section{Conclusiones}

Entre el adulto mayor y la mascota se genera un vínculo lento y progresivo que se fortalece durante las actividades diarias, comparable con el que se establece con un hijo o niño pequeño, que requiere cuidado $\mathrm{y}$ protección.

La compañía de la mascota en todos los espacios y actividades diarias es el aspecto más satisfactorio para los mayores, su presencia permanente les permite tener un interlocutor, jugar, salir y compartir manifestaciones de afecto que evitan la soledad.

Los principales beneficios para la salud son la alegría y felicidad que se generan en el diario compartir con la mascota, así como la paz y tranquilidad para afrontar situaciones de tristeza, ira y angustia, coadyuvantes en la promoción de la salud que trascienden a los demás miembros de la familia

\section{Conflicto de intereses}

Los autores declaran no tener ningún conflicto de intereses.

\section{Referencias}

1. Acero M, Ulloa A (dir). Relación Humano-animal de compañía como un fenómeno sociocultural. Perspectiva para la salud pública [Tesis en internet]. [Bogotá]: Universidad Nacional de Colombia; 2017. Recuperado a partir de: https://repositorio.unal.edu. co/handle/unal/58863

2. Grier KC. Pets in America: A History. North Carolina: UNC Press Books; 2006.

3. Huenchuan S, Rodríguez-Piñero L. Envejecimiento y derechos humanos: situación y perspectivas de protección [Internet]. Santiago de Chile (Chile): Naciones Unidas; 2010. Recuperado a partir de: https://core.ac.uk/download/pdf/38672102.pdf

4. Organización Mundial de la Salud, Organización Panamericana de la Salud. Plan de acción sobre la salud de las personas mayores, incluido el envejecimiento activo y saludable: informe final; Washington (DC): OMS para las Américas; 2019. Report NO.: CD57/INF/9 Recuperado a partir de: https://www3.paho.org/hq/index.php?option=com docman\&view $=$ download\&alias $=49692$-cd57-inf9-s-pda-personas-mayores\&category slug $=\mathrm{cd} 57-$ es\&Itemid $=270 \&$ lang $=\mathrm{es}$

5. Organización Panamericana de la Salud, ICOPE. Guía sobrelaevaluaciónylosesquemasdeatencióncentrados en la persona en la atención primaria de salud [Internet]. Suiza: Organización Panamericana de la Salud; 2020. Recuperado a partir de: https://iris.paho.org/bitstream/ handle/10665.2/51973/OPSFPLHL200004A_spa. pdf? sequence $=5 \&$ isAllowed $=y$

6. Fedesarrollo, Fundación Saldarriaga Concha. Misión Colombia Envejece: cifras, retos y recomendaciones [Internet]. Bogotá (Colombia): Editorial Fundación Saldarriaga Concha; 2015. Recuperado a partir de: $\quad$ https://www.repository.fedesarrollo.org.co/ handle/11445/2724

7. Ministeriode Salud y Protección Social-Departamento Administrativo de Ciencia, Tecnología e Innovación (Colciencias), Universidad del Valle, Universidad de Caldas. Encuesta SABE Colombia: situación de salud, bienestar y envejecimiento en Colombia. Colombia; 2016. 476 p. Disponible en: https://issuu. com/monsafe/docs/sabe colombia - situacion_de person 
8. Ministerio de Salud de Colombia. Boletines poblacionales: Personas Adultas Mayores de 60 Años [Internet]. Bogotá (Colombia): Ministerio de salud y protección social; 2019. [citado el 6 de diciembre de 2020]. Recuperado a partir de: https://www.minsalud. gov.co/sites/rid/Lists/BibliotecaDigital/RIDE/DE/PS/ boletines-poblacionales-envejecimiento.pdf

9. Quintero-Echeverri A, Villamil-Gallego MM, Henao-Villa E, Cardona-Jiménez JE. Diferencias en el sentimiento de soledad entre adultos institucionalizados y no institucionalizados. Rev Fac Nac Salud Pública; 2018; 36(2):49-57. doi: https:// doi.org/10.17533/udea.rfnsp.v36n2a07

10. Müllersdorf M, Granström F, Sahlqvist L, Tillgren P. Aspects of health, physical/leisure activities, work and socio-demographics associated with pet ownership in Sweden. Scand J Soc Med. 2009; 38(1): 53-63. doi: https://doi.org/10.1177/1403494809344358

11. Pikhartova J, Bowling A, Victor C. Does owning a pet protect older people against loneliness? BMC Geriatr. 2014;14(106). doi: https://doi.org/10.1186/1471-2318-14-106

12. Schoenfeld-Tacher R, Kogan LR, Wright ML. Comparison of strength of the human-animal bond between Hispanic and non-Hispanic owners of pet dogs and cats. J Am Vet Med Assoc. 2010; 236 (5): 529-534. https://doi.org/10.2460/javma.236.5.529

13. Morse J. Asuntos críticos en los métodos de investigación cualitativa. Medellín: Publicaciones Universidad de Alicante/Universidad de Antioquia; 2003.

14. Hammersley M, Atkinson P. Etnografía. Métodos de investigación. Barcelona: Paidós; 2001.

15. Strauss A, Corbin J. Bases de la investigación cualitativa: Técnicas y procedimientos para desarrollar la teoría fundamentada. Medellín: Editorial Universidad de Antioquia; 2002. Disponible en: https:// diversidadlocal.files.wordpress.com/2012/09/basesinvestigacion-cualitativa.pdf

16. León MR, Silveira EA, Norman O. Evaluación del conocimiento sobre manejo y cuidado de mascotas en el municipio Santa Clara, Cuba. [Internet] Rev. electrón. vet. 2010; 11(3B):1-12. Recuperado a partir de: https://www.redalyc.org/articulo. oa? idp $=1 \&$ id $=63613140030 \&$ cid $=59698$

17. Carmona Pérez E, Zapata Puerta M, López Pulgarín SE. Familia multiespecie, significados e influencia de la mascota en la familia. Palabra. 2019; 19 (1): $77-$ 90. doi: https://doi.org/10.32997/2346-2884-vol.19num.1-2019-2469

18. Londoño M, Lemos M, Orejuela J. Impacto de la adopción de una mascota en las percepciones de bienestar físico y emocional. Revista de Psicología Universidad de Antioquia. 2018; 10(2): 53-74. doi: https://doi.org/10.17533/udea.rp.v10n2a03
19. Feuerbacher EN, Wynne CD. Shut up and pet me! domestic dogs (canis lupus familiaris) prefer petting to vocal praise in concurrent and single-alternative choice procedures. Behavioural Processes. 2014; 110: 47-59. doi: https://doi.org/10.1016/j.beproc.2014.08.019

20. Hugues B, Álvarez A, Ledón L, Mendoza M, Castelo L, Domínguez E. Percepción de los beneficios de la tenencia de animales de compañía en pacientes con enfermedades cardiovasculares. CorSalud. 2014; 6(1):56-62. https://www.medigraphic.com/cgi-bin/ new/resumen.cgi?IDARTICULO $=56683$

21. Ferreira A. Therapeutic Profit by Companion Animals Employment in Health Care of Older People. Rev. Gerenc. Polit. Salud. 2012; 11(22): 5866. Recuperado a partir de: https://www.researchgate. net/publication/262446750_Therapeutic Profit by_Companion_Animals Employment_in Health Care of Older People

22. Cruz C. Mascotas: ¿Amigos medicinales? Altern. Psicol. 2009; 14(20): 48-57. Recuperado a partir de: http://pepsic.bvsalud.org/scielo.php?script=sci arttext\&pid=S1405-339X2009000100005

23. Custance D, Mayer J. Empathic like responding by domestic dogs (Canis familiaris) to distress in humans: an exploratory study. Anim. Cogn. 2012; 15(5): 851859. doi: https://doi.org/10.1007/s10071-012-0510-1

24. Bowlby J. Una base segura. Aplicaciones clínicas de una teoría del apego. Buenos Aires: Paidós; 2009.

25. Power E. Furry families: making a humandog family through home. Social \& Cultural Geography. 2008; 9(5): 535-555. doi: http://dx.doi. org/10.1080/14649360802217790

26. Martins MF, Pieruzzi, PA, Santos JP, Brunetto MA, Fruchi, VM, Ciari, MB. Grado de apego de los propietarios a los animales de acuerdo con la Escala de Adjuntos a Mascotas de Lexington. Arq. Bras. Med. Vet. Zootec. 2013; 50(5): 64-369. doi: https:// doi.org/10.11606/issn.2318-3659.v50i5p364-369

27. Miralles-Basseda R. Aspectos diagnósticos y terapéuticos. Fragilidad y valoración geriátrica integral. En: Rozman Borstnar C, Cardellach López $\mathrm{F}$, Farreras Rozman, editores. Medicina Interna [Internet]. 18va ed. Barcelona: Elsevier; 2016. Recuperado a partir de: https://www.clinicalkey.es/\#!/ content/book/3-s2.0-B9788490229965001551

28. Calvo P, Bowen J, Bulbena A, Tobeña A, Fatjó J. Highly Educated Men Establish Strong Emotional Links with Their Dogs: A Study with Monash Dog Owner Relationship Scale (MDORS) in Committed Spanish Dog Owners. PLoS ONE. 2016;11(12). doi: https://doi.org/10.1371/journal.pone.0168748

29. Westgarth C, Christley RM, Marvin G, Perkins E. I Walk My Dog Because It Makes Me Happy: A Qualitative Study to Understand Why Dogs Motivate 
Walking and Improved Health. Int. J. Environ. Res. Public. Healt. 2017; 14(8): 936. https://doi. org/10.3390/ijerph14080936

30. Kwong M, Bartholomew K. «Not just a dog»: an attachment perspective on relationships with assistance dogs. Attach. Hum. Dev. 2011;13(5): 42136. doi: https://doi.org/10.1080/14616734.2011.584410

31. Hui GZ, Hill A, Yeung P, Keesing S, Netto JA. Pet ownership and its influence on mental health in older adults. Aging Ment Health. 2019; 24(10): 1605-1612. https://doi.org/10.1080/13607863.2019.1633620

32. Reisbig A, Hafen M, Siqueira A, Girard D, Breunig ZB. Companion Animal Death: A Qualitative Analysis of Relationship Quality, Loss, and Coping. Omega. 2017; 75(2): 124-50. https://doi. org/10.1177/0030222815612607

33. Packman W, Bussolari C, Katz R, Carmack BJ, Field NP. Posttraumatic Growth Following the Loss of a Pet. Omega. 2017; 75(4): 337-59. https://doi. org $/ 10.1177 / 0030222816663411$

34. Ekman P, Oster H. Expresiones faciales de la emoción. Studies in Psychology, 1981; 7 (1): 115-143. https:// doi.org/10.1080/02109395.1981.10821273

35. Taniguchi Y, Seino S, Nishi M, Tomine Y, Tanaka I, Yokoyama Y, Amano H, Kitamura A, Shinkai S. Physical, social, and psychological characteristics of community-dwelling elderly Japanese dog and cat owners. PloS ONE. 2020; 14(3). doi: https://doi. org/10.1371/journal.pone.0229163
36. Jacobs Bao K, Schreer G. Pets and Happiness: Examining the Association between Pet Ownership and Wellbeing, Anthrozoös. 2016; 29 (2): 283-296. doi: http://dx.doi.org/10.1080/08927936.2016.11527 $\underline{21}$

37. Levine, GN, Allen, K., Braun, LT, Christian, HE, Friedmann, E., Taubert, KA, Thomas, SA, Wells, DL y Lange, RA. Pet ownership and cardiovascular risk: a scientific statement from the American Heart Association. Circulación. 2013; 127(23): 2353-2363. doi: https://doi.org/10.1161/CIR.0b013e31829201e1

38. Feng $Z$, Dibben $C$, Witham MD, Donnan PT, Vadiveloo T, Sniehotta F et al. Dog ownership and physical activity in later life: A cross-sectional observational study. Prev. Med. 2014; 66(1): 101-106. doi: https://doi.org/10.1016/j.ypmed.2014.06.004

39. Dall PM, Ellis SLH, Ellis BM, Grant PM, Colyer A, Gee NR, Granat MH, Mills DS. The influence of dog ownership on objective measures of freeliving physical activity and sedentary behaviour in community-dwelling older adults: a longitudinal casecontrolled study. BMC Public Health. 2017; 17(1): 496. doi: https://doi.org/10.1186/s12889-017-4422-5

40. Wood L, Martin K, Christian H, Nathan A, Lauritsen $\mathrm{C}$, Houghton $\mathrm{S}$ et al. El factor mascota: los animales de compañía como un conducto para conocer a las personas, la formación de amistad y el apoyo social. PLoS One. 2015; 10(4): 1-17. https://doi.org/10.1371/ journal.pone. 0122085 